\title{
Anti-Codes in Terms of Berlekamp's Switching Game
}

\author{
Uwe Schauz \\ Department of Mathematics and Statistics \\ King Fahd University of Petroleum and Minerals \\ Dhahran 31261, Saudi Arabia \\ schauz@kfupm.edu.sa \\ Currently at: \\ Abdus Salam School Of Mathematical Sciences \\ GC University, Lahore, Pakistan
}

Submitted: Oct 3, 2011; Accepted: Dec 30, 2011; Published: Jan 6, 2012

Mathematics Subject Classifications: 91A46, 94B05, 51E20

\begin{abstract}
We view a linear code (subspace) $C \leq \mathbb{F}_{q}^{n}$ as a light pattern on the $n$-dimensional Berlekamp Board $\mathbb{F}_{q}^{n}$ with $q^{n}$ light bulbs. The lights corresponding to elements of $C$ are $O N$, the others are $O F F$. Then we allow axis-parallel switches of complete rows, columns, etc. We show that the dual code $C^{\perp}$ contains a vector $v$ of full weight, i.e. $v_{1}, v_{2}, \ldots, v_{n} \neq 0$, if and only if the light pattern $C$ cannot be switched off. Generalizations of this allow us to describe anti-codes with maximal weight $\delta$ in a similar way, or, alternatively, in terms of a switching game in projective space. We provide convenient bases and normal forms to the modules of all light patterns of the generalized games. All our proofs are purely combinatorial and simpler than the algebraic ones used for similar results about anti-codes in $\mathbb{Z}_{k}^{n}$. Aside from coding theory, the game is also of interest in the study of nowhere-zero points of matrices and nowhere-zero flows and colorings of graphs.
\end{abstract}

\section{Introduction}

Berlekamp's Switching Game was invented in the 1960s by Elwyn Berlekamp. The singleplayer game is played on a matrix of $10 \times 10$ light bulbs. An initial light pattern is set up, using $10 \times 10$ individual hidden switches. Then one has to switch off as many lights as possible using 10 row and 10 column switches, which invert the state of each bulb in the corresponding row or column. The smallest possible number of remaining burning lights, if one starts from a worst case initial pattern, is the covering radius of the binary code generated by row and column switches; see, e.g., [FiSl, CaSt, RoVi]. Actually, this binary Berlekamp Code was a main motivation for the study of the game so far. 
In this paper, we will examine a very different connection to coding theory and new game-theoretic questions. We are interested in the question if a given light pattern can be switched off completely or not, since this switchability will turn out to be important in applications. We work in a more general framework, and consider an $n$-dimensional version of Berlekamp's game with $q \times q \times \cdots \times q$ many light bulbs. Here, we restrict $q$ to prime-powers, so that we can identify the light bulbs with the points in the vector space $\mathbb{F}_{q}^{n}$. This will enable us to use some linear algebra arguments. Furthermore, we do not just allow the 2 states $O N$ and $O F F$ for any of the $q^{n}$ light bulbs. It is more general to examine a modulo $r$ version, with $\mathbb{Z}_{r}:=\mathbb{Z} / r \mathbb{Z}$ as the set of possible states of a light bulb. We even allow $r=0$ with the integers $\mathbb{Z}_{0}:=\mathbb{Z}$ as the set of possible states of the points $v \in \mathbb{F}_{q}^{n}$. A (light) patterns is a map $U: \mathbb{F}_{q}^{n} \longrightarrow \mathbb{Z}_{r}$, and an elementary move increases or decreases the state of the bulbs along an axis-parallel affine subspace of dimension $m$. Already the axis-parallel affine $m$-dimensional subspace may be called elementary move or $m$-move. For example, if $(n, q, m, r)=(3,7,2,0)$ then $(*, 5, *):=\left\{(x, 5, y) \mid x, y \in \mathbb{F}_{7}\right\}$ is an elementary 2-move on the 3-dimensional board $\mathbb{F}_{7}^{3}$ over $\mathbb{F}_{7}$. Application of this move transforms a pattern $U: \mathbb{F}_{7}^{3} \longrightarrow \mathbb{Z}_{0}$ either into $U+(*, 5, *)$ or into $U-(*, 5, *)$, as we will write it. So $[U \pm(*, 5, *)](1,5,3)=U(1,5,3) \pm 1$, but $[U \pm(*, 5, *)](1,6,3)=U(1,6,3)$. In Berlekamp's original version of the game $(n, q, m, r)$ was $(2,10,1,2)$. We call the generalized game Berlekamp or Affine Berlekamp modulo $r$ of order $q$ and dimension $n$ with $m$-moves, for short $\mathrm{AB}_{r}^{n, m}\left(\mathbb{F}_{q}\right)$.

Switchability is connected to linear anti-codes. A linear anti-code of length $n$ over $\mathbb{F}_{q}$ is simply a linear code, i.e. a subspace, $U \leq \mathbb{F}_{q}^{n}$. The prefix "anti" just expresses that we are not interested in the minimal weight, but in the maximal weight $\delta$, i.e., the maximum of the weights $\mathrm{w}(u):=|\operatorname{supp}(u)|$ over all elements $u \in U$. Codes with low maximal weight $\delta$ can be used to construct information-theoretically good codes, i.e. codes with big minimal weight, see [MWS, Chapter $17 \S 6]$. We investigate the dual maximal weight, which would be the maximal weight of the dual (orthogonal) code $U^{\perp}$. Our discovery is that, if we view a subspace $U \leq \mathbb{F}_{q}^{n}$ as a $0-1$ pattern on the board $\mathbb{F}_{q}^{n}$, $U$ can be switched off with $m$-moves if and only if its dual maximal weight is at most $n-m$. More precisely, it will turn out that $U$ can be switched off modulo $r$ if and only if it can be switched off modulo any other $r^{\prime}$, provided that $r$ and $r^{\prime}$ do not divide $|U|$. We just use the term $m$-switchability when we refer to any of these equivalent cases, including the case $r=0$.

We hope that the new connection from anti-codes to Berlekamp's Game will lead to new insights about liner codes, and that the game can become a useful tool in codingtheory. One case of particular interest was already investigated in our paper [Sch1]. There, we examined the existence of full weight vectors in codes that arise from graph theory. Using the connection to Berlekamp's Game, we saw that a graph $G$ has a nowhere-zero $k$-flow if and only if the $\mathbb{Z}_{k}$-bond space of $G$ cannot be switched off. The graph $G$ has a vertex coloring with $k$ colors if and only if a certain corresponding code over $\mathbb{Z}_{k}$ cannot be switched off. Similar statements could be proven for Tait colorings and for nowherezero points of matrices. We also introduced normal forms to equivalence classes of light patterns, and obtained new equivalents for the existence of full weight vectors in $U^{\perp}$. This 
led to new equivalents, e.g., for the Four Color Problem, Tutte's Flow Conjectures and Jaeger's Conjecture. Two of our equivalents for colorability and existence of nowhere zero flows of graphs included as special cases results by Matiyasevich, by Balázs Szegedy and by Onn. Alon and Tarsi's sufficient condition for vertex colorability also arose, remarkably, as a generalized full equivalent. In our present paper, we do not deal with such graphtheoretic problems, and there are also three main differences on the game-theoretic side:

1. Here, we work over $\mathbb{F}_{q}^{n}$ as board, which requires $q$ to be a prime-power. In [Sch1], we worked over the board $\mathbb{Z}_{k}^{n}$, with no further restriction on $k$.

2. In the present paper, we present simple combinatorial proofs. In [Sch1], over the rings $\mathbb{Z}_{k}^{n}$, more complicated algebraic proofs where required.

3. In [Sch1], we restricted ourselves to the case $m=1$. The treatment of the general case would have been possible, but would have made the algebraic proofs there even more complicated.

Based on our study of the vector space $\mathbb{F}_{q}^{n}$ as board of the game, we may also wonder if the game can be transferred to finite projective spaces, with one light bulb at any projective point. We are only interested in the switchability of subspaces $U$ of $\mathbb{F}_{q}^{n}$, and they correspond to subspaces $U^{1}$ of $\mathrm{PG}_{n-1}\left(\mathbb{F}_{q}\right):=\mathrm{PG}\left(\mathbb{F}_{q}^{n}\right)$. Therefore, one might expect that it is straight forward to find an equivalent game in projective space. However, our moves in $\mathrm{AB}_{r}^{n-1, m}\left(\mathbb{F}_{q}\right)$ are usually not linear subspaces, and in the construction of $\mathrm{PG}_{n-1}$ out of $\mathbb{F}_{q}^{n}$ one just discards the zero $0 \in \mathbb{F}_{q}^{n}$. Therefore, on one hand, there are some difficulties in constructing a projective equivalent, but, on the other hand, if the game on $\mathrm{PG}_{n-1}\left(\mathbb{F}_{q}\right)$ just would be a straight copy of the game on $\mathbb{F}_{q}^{n}$, it would not be very interesting. We will work out a simple and nice definition of the allowed moves in Projective Berlekamp. Our projective $m$-moves will be switches of subspaces of $\mathrm{PG}_{n-1}\left(\mathbb{F}_{q}\right)$ that run through at least $m$ of the $n$ coordinate axes $\left\langle e_{1}\right\rangle,\left\langle e_{2}\right\rangle, \ldots$, $\left\langle e_{n}\right\rangle$, viewed as independent projective points in $\mathrm{PG}_{n-1}\left(\mathbb{F}_{q}\right)$. Over finite fields $\mathbb{F}_{q}$ with more then 2 elements, $q>2$, our new Projective Berlekamp $\mathrm{PB}_{r}^{n-1, m}\left(\mathbb{F}_{q}\right)$ will turn out to be an equivalent to Affine Berlekamp $\mathrm{AB}_{r}^{n-1, m}\left(\mathbb{F}_{q}\right)$. Hence, $U^{1}$ can be switched off in $\mathrm{PB}_{r}^{n-1, m}\left(\mathbb{F}_{q}\right)$ if and only if $U^{\perp}$ has maximal weight at most $n-m$.

With the described connections to anti-codes, our Berlekamp Games have the potential to become a helpful tool in coding theory and combinatorics. At least, we think that they are simple enough to be attractive. One thing that has already proven to be useful, are normal forms to the equivalence classes of switchable patterns. In [Sch1], we even provided some formulas to calculate them (for $m=1$ ). In this paper, we do not look at these formulas, but we present generalized normal forms for arbitrary $m$. Also of interest may be the connection to the Combinatorial Nullstellensatz, which can be proven using a kind of Berlekamp Game, see [Sch2, Section 7]. The connection to the polynomial manipulation techniques in this paper was, in fact, the starting idea behind our first paper about Berlekamp's Game [Sch1].

We formally introduce Affine Berlekamp in the next section, Section 2. In Section 3, we work out the connections to coding theory. This study of linear subspaces (linear 
codes), as light patterns, will lead the way to projective spaces and Projective Berlekamp, which we examine in Section 4.

\section{Affine Berlekamp}

We start here with a more general situation than described in the introduction. We take any finite set $I$ (of light bulbs) as board, and any system $\mathcal{M} \subseteq \mathbb{Z}_{r}^{I}$ of (light) patterns (i.e. maps $U: I \longrightarrow \mathbb{Z}_{r}$ ) as our collection of elementary moves:

Definition 2.1 (General Berlekamp). A pair $(I, \mathcal{M})$ of a finite set $I$ and a system $\mathcal{M} \subseteq \mathbb{Z}^{I}$ of patterns is a (General) Berlekamp on the board $I$. The elements of $\mathcal{M}$ are its (elementary) moves. The elements of its $\mathbb{Z}$-linear span $\langle\mathcal{M}\rangle \leq \mathbb{Z}^{I}$ are its switchable patterns or composed moves, they can be switched off by a sequence of moves. By replacing $\mathbb{Z}=: \mathbb{Z}_{0}$ with $\mathbb{Z}_{r}:=\mathbb{Z} / r \mathbb{Z}$, we obtain $(I, \mathcal{M})_{r}$, (General) Berlekamp modulo $r$.

$\langle\mathcal{M}\rangle, \mathbb{Z}_{r}$

$(I, \mathcal{M})_{r}$

In what follows, we mostly work over $\mathbb{Z}_{0}=\mathbb{Z}$ and may interpret the results modulo $r$ afterwards. We identify subsets $U \subseteq I$ with their characteristic functions $I \longrightarrow\{0,1\}$ as $0-1$ light patterns, i.e.,

$$
U(v):= \begin{cases}1 & \text { if } v \in U, \\ 0 & \text { if } v \notin U .\end{cases}
$$

This is used extensively. It simplifies notation, but can lead to unusual expressions. For example, the one-point sets $\{v\}(v \in I)$ are also viewed as $0-1$ patterns

$$
\{v\}: I \longrightarrow\{0,1\}, u \longmapsto\{v\}(u) \text {. }
$$

These one-point sets form the standard basis of $\mathbb{Z}^{I}$.

Based on General Berlekamp we can now introduce Affine Berlekamp, and later Projective Berlekamp. For Affine Berlekamp on boards of the form $I:=I_{1} \times I_{2} \times \cdots \times I_{n}$ or $I:=\mathbb{Z}_{k}^{n}$ see [Sch1]. Here we only study the $n$-dimensional $q \times q \times \cdots \times q$ board $I:=\mathbb{F}_{q}^{n}$. This board carries the structure of a vector space, an $\mathbb{F}_{q}$-modulo. Since the set of light patterns $\mathbb{Z}^{I}$ is also a module, this time over $\mathbb{Z}$ as ring of scalars, we have to be careful with the notation. Subsets of $I$ are usually viewed as 0-1 patterns in $\mathbb{Z}^{I}$ and added in $\left(\mathbb{Z}^{I},+\right)$, while elements of $I=\mathbb{F}_{q}^{n}$ or a combination of one element and one subset of $\mathbb{F}_{q}^{n}$ are always added in $\left(\mathbb{F}_{q}^{n},+\right)$, e.g. $(0,1)+(1,0)=(1,1)$ but $\{(0,1)\}+\{(1,0)\}=\{(0,1),(1,0)\}$. Similar rules are used for the two scalar multiplications. The two linear spans are denoted differently. We use $\langle\ldots\rangle$ in the module $\mathbb{Z}^{I}$ and $\langle\ldots\rangle$ in the vector space $\mathbb{F}_{q}^{n}$. With this notational basis, we define:

Definition 2.2 (Affine Berlekamp). We write $\mathrm{AB}^{n, m}=\mathrm{AB}_{0}^{n, m}\left(\mathbb{F}_{q}\right)$ for the Berlekamp $\left(\mathbb{F}_{q}^{n}, \mathcal{A M}_{m}\right)$ - called Affine Berlekamp with m-moves - where $\mathcal{A M}_{m}$ is the set of all affine axis-parallel $m$-dimensional subspaces $v+\left\langle e_{j_{1}}, e_{j_{2}}, \ldots, e_{j_{m}}\right\rangle$ of $\mathbb{F}_{q}^{n}$. In the modulo $r$ case, with $\mathbb{Z}_{r}:=\mathbb{Z} / r \mathbb{Z}$ in the place of $\mathbb{Z}=: \mathbb{Z}_{0}$, we write $\mathrm{AB}_{r}^{n, m}$ with $r$ as index. 
We also provide a simpler notation for moves. If $v \in \mathbb{F}_{q}^{n}$ and $J \subseteq\{1,2, \ldots, n\}$, we set

$$
v\left\lceil J:=v+\left\langle e_{J}\right\rangle \quad \text { where } \quad e_{J}:=\left\{e_{j} \mid j \in J\right\} .\right.
$$

For example, an elementary 3-move trough a point $v=\left(v_{1}, v_{2}, \ldots, v_{6}\right)$ in $\mathbb{F}_{q}^{6}$ parallel to 3 axes $\left\langle e_{j}\right\rangle$, say $j \in J:=\{2,4,5\}$, may be written as

$$
v\left\lceil J=\left(v_{1}, *, v_{3}, *, *, v_{6}\right):=\left\{v_{1}\right\} \times \mathbb{F}_{q} \times\left\{v_{3}\right\} \times \mathbb{F}_{q} \times \mathbb{F}_{q} \times\left\{v_{6}\right\} .\right.
$$

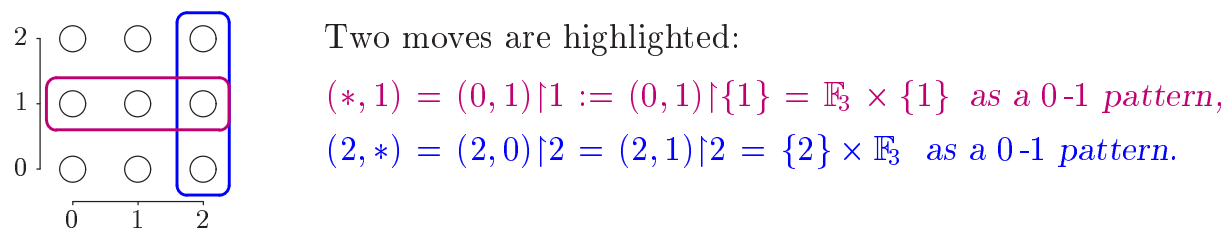

Figure 1: $\mathrm{AB}^{2,1}\left(\mathbb{F}_{3}\right)$

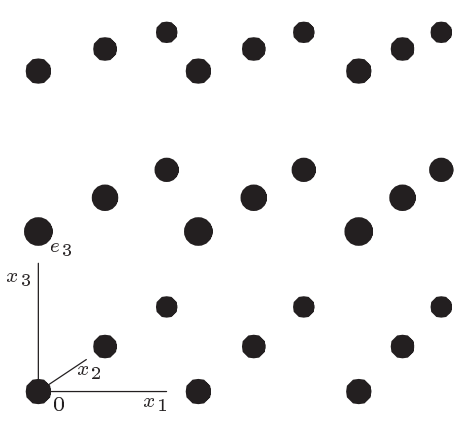

Figure 2: The basis vector $B_{0, e_{3}}:=$ $e_{3}\left\lceil\operatorname{ker}\left(e_{3}\right)=(0,0,1)\left\lceil\{1,2\}\right.\right.$ over $\mathbb{F}_{3}^{3}$

The patterns

$$
B_{0, v}:=v\left\lceil\operatorname{ker}(v) \text { with } \operatorname{ker}(v):=\left\{j \mid v_{j}=0\right\}=:\{1,2, \ldots, n\} \backslash \operatorname{supp}(v),\right.
$$

where $v=\left(v_{1}, v_{2}, \ldots, v_{n}\right)$ runs through $\mathbb{F}_{q}^{n}$ (see Figure 2), form a basis $\mathcal{B}_{0}$ of the module of all light patterns $\mathbb{Z}^{\mathbb{E}^{n}}$. Indeed, the change of bases matrix from $\mathcal{B}_{0}$ to the standard basis $\left\{\{v\}: v \in \mathbb{F}_{q}^{n}\right\}$ is triangular with ones on the diagonal. We just have to choose a suitable linear ordering on the set $\mathbb{F}_{q}^{n}$ of indices of the two bases, in order to turn them into appropriately ordered bases. We do this by selecting the linear order $<$ on $\mathbb{F}_{q}^{n}$ such that the weight function $\mathrm{w}: v \longmapsto w(v)=n-|\operatorname{ker}(v)|$ becomes a monotonous decreasing function, i.e.

$$
\mathrm{w}(v)>\mathrm{w}\left(v^{\prime}\right) \Longleftrightarrow|\operatorname{ker}(v)|<\left|\operatorname{ker}\left(v^{\prime}\right)\right| \Longrightarrow v<v^{\prime} .
$$

In this way, $B_{0, v}=v \uparrow \operatorname{ker}(v)$ only contains elements $u$ that come before $v(u \leq v)$, ensuring zeros below/above the diagonal. We even see that, for any $k \leq n$,

$$
\left.\left\langle\mathcal{B}_{0}^{\leq k}\right\rangle=\langle\{v\}| v \in \mathbb{F}_{q}^{n} \text { with }|\operatorname{ker}(v)| \leq k\right\rangle
$$


where

$$
\mathcal{B}_{0}^{\leq k}:=\left\{B_{0, v}: v \in \mathbb{F}_{q}^{n} \text { with }|\operatorname{ker}(v)| \leq k\right\} .
$$

This basis has the advantage that it contains a basis of the submodule of all $m$-switchable light patterns,

$$
\left\langle\mathcal{B}_{0}^{\geq m}\right\rangle=\left\langle\mathcal{A} \mathcal{M}_{m}\right\rangle
$$

where $\mathcal{B}_{0}^{\geq m}$ is analogues to $\mathcal{B}_{0}^{\leq k}$ above. This is easily verified: To prove the inclusion $\left\langle\mathcal{B}_{0}^{>m}\right\rangle \supseteq\left\langle\mathcal{A M}_{m}\right\rangle$, let $v\left\lceil J \in \mathcal{A M}_{m}\right.$. We may assume $|J|=m>0$ and $n \in J$ as the case $m=0$ is trivial. Now let $v^{\prime}:=\left(v_{1}, v_{2}, \ldots, v_{n-1}\right)$, then $v^{\prime} \uparrow(J \backslash n)$ is an $(m-1)$-dimensional move on the $(n-1)$-dimensional board. Using an induction argument, we realize that this move is a linear combination of certain $B_{0,\left(u_{1}, \ldots, u_{n-1}\right)}$ with at least $m-1$ zero entrees $u_{j}$. The corresponding linear combination of the extended $B_{0,\left(u_{1}, \ldots, u_{n-1}, 0\right)} \in$ $\mathcal{B}_{0}^{\geq m}$ is then equal to $v\left\lceil J\right.$, so that $\left\langle\mathcal{B}_{0}^{\geq m}\right\rangle \supseteq \mathcal{A M}_{m}$. The opposite inclusion can be proven similarly. Actually, any axis-parallel affine subspaces of dimension at least $m$ is $m$-switchable, and this is just a special case of our Lemma 3.1 further below.

The existence of a basis with the described properties has an important consequence:

Theorem 2.3. The $\mathbb{Z}$-submodule of all switchable patterns in $\mathrm{AB}^{n, m}\left(\mathbb{F}_{q}\right)$ is saturated, i.e., its elementary divisors are units. In particular, if the multiple $z U: v \longmapsto z U(v)$ ( $z \in \mathbb{Z} \backslash 0$ ) of a pattern $U$ can be switched off, then $U$ can be switched off.

Our two-tier basis $\mathcal{B}_{0}=\mathcal{B}_{0}^{<m} \uplus \mathcal{B}_{0}^{>m}$ also gives rise to a normal form $N_{0}^{m}: U \longmapsto$ $N_{0}^{m}(U)$ to the equivalence classes of patterns $U \in \mathbb{Z}^{\mathbb{F}_{q}^{n}}$, where two patterns are equivalent if there is a sequence of $m$-moves that transforms one into the other:

Theorem 2.4. The $\mathbb{Z}$-submodule $\langle\{v\}|| \operatorname{ker}(v)|\langle m\rangle=\left\langle\mathcal{B}_{0}^{<m}\right\rangle \leq \mathbb{Z}^{\mathbb{F}_{q}^{n}}$ of all those patterns $U: \mathbb{F}_{q}^{n} \longrightarrow \mathbb{Z}$ which are zero on all $(n-m)$-dimensional coordinate subspaces $\left\langle e_{j_{1}}, e_{j_{2}}, \ldots, e_{j_{n-m}}\right\rangle \leq \mathbb{F}_{q}^{n}$, is a complement to the $\mathbb{Z}$-submodule of all m-switchable patterns $\left\langle\mathcal{A} \mathcal{M}_{m}\right\rangle=\left\langle\mathcal{B}_{0}^{\geq m}\right\rangle$

$$
\left\langle\{v\}_{1}|\operatorname{ker}(v)|<m\right\rangle \oplus\left\langle\mathcal{A} \mathcal{M}_{m}\right\rangle=\mathbb{Z}^{\mathbb{F}_{q}^{n}}
$$

In particular, $\left\langle\{v\}_{1}|\operatorname{ker}(v)|<m\right\rangle$ is a set of representatives $N_{0}^{m}(U)$ to the equivalence classes $U+\left\langle\mathcal{A M}_{m}\right\rangle \in \mathbb{Z}^{\mathbb{F}_{q}^{n}} /\left\langle\mathcal{A M}_{m}\right\rangle$ of patterns $U \in \mathbb{Z}^{\mathbb{F}_{q}^{n}}$ with respect to m-switches.

If we want to transform a pattern $U: x \longmapsto U(x)$ into its normal form $N_{0}^{m}(U)$, there is an easy way to do so. Just select a point $x \in \operatorname{supp}(U)$ with maximal $|\operatorname{ker}(x)|$ and then switch (increase) $B_{0, x}$ exactly $-U(x)$ many times, provided that $|\operatorname{ker}(x)| \geq m$ so that $B_{0, x}$ is $m$-switchable. (If $-U(x)<0$ this means to decrease $B_{0, x}$ exactly $U(x)$ many times.) Afterwards, $U(x)=0$ and, aside from this value, only values $U(y)$ with $|\operatorname{ker}(y)|<|\operatorname{ker}(x)|$ have changed. We have eliminated $x$ from $\operatorname{supp}(U)$ without adding points $y$ that are "as bad as $x$ ". Repeating this step as long as possible, we will finally reach a cleared out $U$ with $|\operatorname{ker}(x)|<m$ for all $x \in \operatorname{supp}(U)$, i.e. the initial $U$ was transformed into its normal form in $\langle\{v\}|| \operatorname{ker}(v) \mid<m\rangle$. 
If we look a bit closer to what happens in each modification step of this procedure, we see that only values $U(y)$ with $\left.y\right|_{\operatorname{supp}(x)}=\left.x\right|_{\operatorname{supp}(x)}$ are modified. So, if we start with a single point pattern $U:=\{u\}$, any subsequently switched light $y$ coincides with $u$ on $\operatorname{supp}(u), y_{j}=u_{j}$ for all $j$ with $u_{j} \neq 0$. From this we can deduce the following lemma, which we will need in the section about Projective Berlekamp, Section 4:

Lemma 2.5. For any $u \in \mathbb{F}_{q}^{n}$, we have $\operatorname{supp}\left(N_{0}^{m}(\{u\})\right) \subseteq u \uparrow \operatorname{ker}(u)$. In other words, if $u_{j} \neq 0$ for $a j \in\{1,2, \ldots, n\}$, then all $x \in \operatorname{supp}\left(N_{0}^{m}(\{u\})\right)$ fulfill $x_{j}=u_{j}$.

\section{Switchable Codes}

This section describes the connection between the maximal dual weight of a code $U \leq \mathbb{F}_{q}^{n}$, i.e. the maximal weight $\mathrm{w}(v)$ of elements $v$ of $U^{\perp}:=\{v:(v \cdot u)=0$ for all $u \in U\}$, and the $m$-switchability of $U$ as $0-1$ pattern in $\operatorname{AB}_{r}^{n, m}\left(\mathbb{F}_{q}\right)$. We will need the simple observation that the $m$-switchable subspaces of $\mathbb{F}_{q}^{n}$ form a filter in the subspace lattice of $\mathbb{F}_{q}^{n}$ :

Lemma 3.1. If an subspace $U \leq \mathbb{F}_{q}^{n}$, as 0-1 pattern in $\mathrm{AB}_{r}^{n, m}\left(\mathbb{F}_{q}\right)$, contains an $m$-switchable subspace, then it is m-switchable itself. In particular, this holds for the coordinate subspaces $\left\langle e_{j_{1}}, e_{j_{2}}, \ldots, e_{j_{m}}\right\rangle, j_{1}<j_{2}<\cdots<j_{m}$ :

$$
e_{j_{1}}, e_{j_{2}}, \ldots, e_{j_{m}} \in U \quad \Longrightarrow \quad U \text { is m-switchable. }
$$

Proof. Let $W$ be the $m$-switchable subspace of $U$, then $U$ can be decomposed into affine subspaces of the form $u+W$. Since these shifted copies of $W$ are still $m$-switchable, $U$ can be switched off piecemeal.

We will also need the following well-known fact:

Lemma 3.2. Let $U<\mathbb{F}_{q}^{n}$ be a proper subspace of codimension $s:=n-\operatorname{dim}(U)$. Then $U$ is contained in $q^{s-1}+q^{s-2}+\cdots+q^{0}$ many hyperplanes $H \geq U$, and each point $v \in \mathbb{F}_{q}^{n} \backslash U$ outside of $U$ is contained in $q^{s-2}+q^{s-3}+\cdots+q^{0}$ many of them. In particular,

$$
q^{s-1} U+\left(q^{s-2}+\cdots+q^{0}\right) \mathbb{F}_{q}^{n}=\sum\{H \geq U \mid H \text { is hyperplane }\} .
$$

Based on these lemmas, we can now prove our core theorem:

Theorem 3.3. For subspaces $U \leq \mathbb{F}_{q}^{n}, m \in\{1,2, \ldots, n-1\}$, and $r \in\{2,3,4, \ldots\}$ not dividing $|U|$, the following are equivalent:

(i) $U^{\perp}$ has maximal weight at most $n-m$.

(ii) $U$ can be switched off with m-moves.

(iii) $U$ can be switched off modulo $r$ with m-moves. 
Proof. The implication $(i i) \Rightarrow($ iii $)$ is trivial. In order to prove $($ iii $) \Rightarrow(i)$, assume that $U^{\perp}$ contains a vector $f$ with less than $m$ zero entrees $f_{j}=0$. Obviously, $f^{\perp}$ contains $U$ and is disjoint to the hyperplane $f_{0}+f^{\perp}$, where we are choosing $f_{0} \notin f^{\perp}$. In particular, our initial light pattern $U$ has $|U|$ many burning lights in $f^{\perp}$, and none in $f_{0}+f^{\perp}$. This makes a difference of $|U|$,

$$
\sum_{v \in f^{\perp}} U(v)-\sum_{v \in f_{0}+f^{\perp}} U(v)=|U| \not \equiv 0 \quad(\bmod r) .
$$

If we now perform an elementary $m$-dimensional switch $v\left\lceil\left\{j_{1}, j_{2}, \ldots, j_{m}\right\}\right.$, each of the two sums in this difference changes by $\pm q^{m-1}$, since the hyperplane $f^{\perp}$ is not parallel to $m$ many coordinate axes $\left\langle e_{j}\right\rangle$ at a time, as

$$
\left(f \cdot e_{j}\right)=f_{j}=0 \text { for less then } m \text { indices } j .
$$

Therefore, the difference will not change, even if we perform a whole sequence of elementary moves. It is invariant and will never become zero. In particular, it is not possible to switch off all lights.

It is left to prove $(i) \Rightarrow(i i)$. The case $U=\mathbb{F}_{q}^{n}$ is trivial, $\mathbb{F}_{q}^{n}$ is always switchable. If $U^{\perp}>\{0\}$ has maximal weight at most $n-m$, then every hyperplane $H \geq U$ also has the property that its orthogonal space $H^{\perp} \leq U^{\perp}$ has maximal weight at most $n-m$. Hence, if $H^{\perp}=\left\langle\left(f_{1}, f_{2}, \ldots, f_{n}\right)\right\rangle$, then $f_{j}=0$ for (at least) $m$ indices $j$, say $j_{1}, j_{2}, \ldots, j_{m}$, and this means that $e_{j_{1}}, e_{j_{2}}, \ldots, e_{j_{m}} \in H$. Therefore, by Lemma 3.1, $H$ can be $m$-switched, i.e., each hyperplane $H \geq U$ can be $m$-switched. However, by Lemma 3.2, $q^{\operatorname{codim}(U)-1} U$ is basically the sum of these hyperplanes; only the full space $\mathbb{F}_{q}^{n}$ has to be subtracted several times. Therefore, $q^{\operatorname{codim}(U)-1} U$ is switchable, and this implies that $U$ is switchable, as, by Theorem 2.3 , the $\mathbb{Z}$-submodule of all switchable patterns is saturated.

This theorem also follows from the following somehow interesting observation, for which we have currently no further application:

Theorem 3.4. For given $0<m \leq n$, set $c_{0}:=1, c_{1}, c_{2}, \ldots, c_{m-1}:=0$ and recursively define $c_{i}:=-\sum_{j=0}^{i-1} c_{j}\left(\begin{array}{c}i \\ j\end{array}\right)$ for $i=m, m+1, \ldots, n$. Hence, for $m=1,\left(c_{i}\right)=\left((-1)^{i}\right)$.

Let $U \leq \mathbb{F}_{q}^{n}$ be a subspace with dual maximal weight at most $n-m$. Then $q^{\operatorname{codim}(U)} U$ can be switched off by adding for each subset $E \subseteq\left\{e_{1}, e_{2}, \ldots, e_{n}\right\}$ with $|E| \geq m$ the $m$-switchable subspace $\langle U \cup E\rangle$ exactly $c_{|E|} q^{\operatorname{codim}\langle U \cup E\rangle}$ many times, followed by switching the full board $\mathbb{F}_{q}^{n}$ a multiple of $q$ many times. More formally,

$$
\sum_{E \subseteq\left\{e_{1}, \ldots, e_{n}\right\}} c_{|E|} q^{\operatorname{codim}\langle U \cup E\rangle}\langle U \cup E\rangle \equiv \emptyset \quad\left(\bmod q \mathbb{F}_{q}^{n}\right)
$$

where the summand with $E=\emptyset$ is the initial pattern $U$, those with $0<|E|<m$ have vanishing coefficients, and those with $|E| \geq m$ are trivially $m$-switchable.

Proof. We may assume $U<\mathbb{F}_{q}^{n}$, as the case $m=n$ is trivial. Since $U$ has dual maximal weight at most $n-m$, every hyperplane $H \geq U$ has dual maximal weight at most $n-m$ 
as well. Hence, if $H=\left(f_{1}, f_{2}, \ldots, f_{n}\right)^{\perp}$, then $\left(f_{1}, f_{2}, \ldots, f_{n}\right)$ has maximal weight at most $n-m$, and $f_{j}=0$ for at least $m$ many indices $j \in\{1,2, \ldots, n\}$. This means that

$$
i:=|\mathcal{E} \cap H| \geq m, \text { where } \mathcal{E}:=\left\{e_{1}, e_{2}, \ldots, e_{n}\right\} .
$$

Hence, the recursive definition of $c_{i}$ applies and

$$
\sum_{E \subseteq \mathcal{E} \cap H} c_{|E|}=c_{i}+\sum_{j=0}^{i-1} c_{j}\left(\begin{array}{l}
i \\
j
\end{array}\right)=0 \quad \text { for all } H \in \mathcal{H}_{U}:=\{H \geq U: H \text { is hyperplane }\},
$$

so that, by Lemma 3.2 ,

$$
\sum_{E \subseteq \mathcal{E}} c_{|E|} q^{\operatorname{codim}\langle U \cup E\rangle}\langle U \cup E\rangle \stackrel{\left(q \mathbb{F}_{q}^{n}\right)}{\equiv} \sum_{E \subseteq \mathcal{E}} c_{|E|} \sum_{\substack{H \in \mathcal{H}_{U} \\ H \supseteq E}} q H=\sum_{H \in \mathcal{H}_{U}}\left[\sum_{\substack{E \subseteq \mathcal{E} \\ E \subseteq H}} c_{|E|}\right] q H=\emptyset .
$$

\section{Projective Berlekamp}

We want to introduce Projective Berlekamp in a way that describes the connection to Affine Berlekamp as directly as possible, so that we can easily transfer results from one game to the other. For this reason, we will have to study homotety-invariant patterns in Affine Berlekamp, i.e. patterns $U: \mathbb{F}_{q}^{n} \longrightarrow \mathbb{Z}$ with the property

$$
\mathrm{M}_{\lambda} U=U \text { for all } \lambda \in \mathbb{F}_{q} \backslash 0 \text {, }
$$

where

$$
\mathrm{M}_{\lambda} U(\lambda v):=U(v) \text { for all } v \in \mathbb{F}_{q}^{n} .
$$

Such patterns $U$ can easily be viewed as patterns $U^{1}$ on the projective board $\mathrm{PG}_{n-1}\left(\mathbb{F}_{q}\right)$. Just assign to the projective point $\langle v\rangle \neq\{0\}$ the value $U^{1}(\langle v\rangle):=U(v)$. However, what shall happen with the value $U(0)$ of $0 \in \mathbb{F}_{q}^{n}$ ? Well, for the time being, let us try to loose no information. Let us introduce in Projective Berlekamp an additional light, the counter, corresponding to the 0 in $\mathbb{F}_{q}^{n}$. We represent this counter by the subset $\{0\} \subseteq \mathbb{F}_{q}^{n}$, so that we can define an extended projective board $\mathrm{PGC}_{n-1}\left(\mathbb{F}_{q}\right)$ as the set

$$
\operatorname{PGC}_{n-1}\left(\mathbb{F}_{q}\right):=\left\{\langle v\rangle \mid v \in \mathbb{F}_{q}^{n}\right\},
$$

and define the projective copy $U^{1}: \mathrm{PGC}_{n-1}\left(\mathbb{F}_{q}\right) \longrightarrow \mathbb{Z}$ of a homotety-invariant pattern $U: \mathbb{F}_{q}^{n} \longrightarrow \mathbb{Z}$ via

$$
U^{1}(\langle v\rangle):=U(v) \text { for all } v \in \mathbb{F}_{q}^{n} \text {, including } v=0 .
$$

If the homotety-invariant pattern $U$ is given as a subset, respectively a subspace, of $\mathbb{F}_{q}^{n}$, then we also call $U^{1}$ a subset, respectively a subspace, of $P G C_{n-1}\left(\mathbb{F}_{q}\right)$. Our definition describes a bijection between homotety-invariant patterns in Affine Berlekamp $\mathrm{AB}_{r}^{n, m}$ and 
patterns in Projective Berlekamp with counter, $\mathrm{PBC}_{r}^{n-1, m}$, as we will write. However, the moves also should go well together. The moves in Affine Berlekamp do not run through the point of origin 0 in general, but we will show that we can restrict ourselves to moves that are vector subspaces of $\mathbb{F}_{q}^{n}$. With this insight, it will be straight forward to define the moves in $\mathrm{PBC}_{r}^{n-1, m}$ and its version $\mathrm{PB}_{r}^{n-1, m}$ without counter. Several examinations are required to reach this point. We start with the following simple lemma:

Lemma 4.1. For $\left(c_{v}\right) \in \mathbb{Z}^{\mathbb{F}_{q}^{n}}$ and $\lambda \in \mathbb{F}_{q} \backslash 0$ holds:

$$
U=\sum_{v \in \mathbb{F}_{q}^{n}} c_{v} B_{0, v} \quad \Longrightarrow \quad \mathrm{M}_{\lambda} U=\sum_{v \in \mathbb{F}_{q}^{n}} c_{\lambda^{-1} v} B_{0, v}
$$

Proof. We prove this pointwise:

$$
\mathrm{M}_{\lambda} U(\lambda u) \stackrel{\text { def }}{=} U(u)=\sum_{v \in \mathbb{F}_{q}^{n}} c_{v} B_{0, v}(u)=\sum_{v \in \mathbb{F}_{q}^{n}} c_{v} B_{0, \lambda v}(\lambda u)=\left[\sum_{w \in \mathbb{F}_{q}^{n}} c_{\lambda^{-1} w} B_{0, w}\right](\lambda u) .
$$

With this we can prove the following version of our Theorems 2.3 and 2.4 for homotetyinvariant patterns:

Theorem 4.2. The pattern $\bar{B}_{\langle 0\rangle}:=B_{0,0}=\mathbb{F}_{q}^{n}$ together with the $\frac{q^{n}-1}{q-1}$ many patterns

$$
\bar{B}_{\langle v\rangle}:=\sum_{\lambda \in \mathbb{F}_{q} \backslash 0} B_{0, \lambda v}=\left\langle\{v\} \cup e_{\operatorname{ker}(v)}\right\rangle-\left\langle e_{\operatorname{ker}(v)}\right\rangle \quad \text { with }\langle v\rangle \in \mathrm{PG}_{n-1}\left(\mathbb{F}_{q}\right)
$$

form a basis $\overline{\mathcal{B}}$ of the module $\mathbb{Z}^{\mathbb{F}_{q}^{n}}$ of all homotety-invariant patterns over $\mathbb{F}_{q}^{n}$.

The $\mathbb{Z}$-submodule of $m$-switchable homotety-invariant patterns on $\mathbb{F}_{q}^{n}$ is spanned by $\overline{\mathcal{B}}^{\geq m}:=\left\{\bar{B}_{\langle v\rangle} \mid v \in \mathbb{F}_{q}^{n}\right.$ with $\left.|\operatorname{ker}(v)| \geq m\right\}$. A complement, inside the $\mathbb{Z}$-module of homotety-invariant patterns, is given by $\langle\langle v\rangle \backslash 0|| \operatorname{ker}(v) \mid<m\rangle$, which is also spanned by $\overline{\mathcal{B}}^{<m}:=\overline{\mathcal{B}} \backslash \overline{\mathcal{B}} \geq m$.

Proof. The linear independence of $\overline{\mathcal{B}}$ follows from the fact that the different $\bar{B}_{\langle v\rangle}$ bundle together disjoint sets of base vectors $B_{0, \lambda v}$, as $\langle v\rangle \backslash 0 \cap\langle w\rangle \backslash 0=\emptyset$ if $\langle v\rangle \neq\langle w\rangle$. To verify the generating property, let $U=\sum_{v} c_{v} B_{0, v}$ be homotety-invariant, then $c_{\lambda v}=c_{v}$ for all $v \in \mathbb{F}_{q}$ and $\lambda \in \mathbb{F}_{q} \backslash 0$, by Lemma 4.1, so that we may define $\bar{c}_{\langle v\rangle}:=c_{v}$ for all $v \in \mathbb{F}_{q}^{n}$. With this definition,

$$
U=c_{0} B_{0,0}+\sum_{\langle v\rangle \in \mathrm{PG}_{n-1}} \sum_{w \in\langle v\rangle \backslash 0} c_{w} B_{0, w}=\bar{c}_{0} \bar{B}_{\langle 0\rangle}+\sum_{\langle v\rangle \in \mathrm{PG}_{n-1}} \bar{c}_{\langle v\rangle} \bar{B}_{\langle v\rangle}
$$

which shows that our basis generates all homotety-invariant patterns. Moreover, if $U$ is $m$-switchable, i.e., if all coefficients $c_{w}$ with $|\operatorname{ker}(w)|<m$ are zero, then all $\bar{c}_{\langle v\rangle}$ with $|\operatorname{ker}(v)|<m$ are zero as well, and $U \in\langle\overline{\mathcal{B}} \geq m\rangle$. Hence, $\overline{\mathcal{B}} \geq m$ spans the whole submodule of $m$-switchable homotety-invariant patterns, but also not more. It obviously has $\left\langle\overline{\mathcal{B}}^{<m}\right\rangle$ as complement, which is equal to $\langle\langle v\rangle \backslash 0|| \operatorname{ker}(v) \mid<m\rangle$, in analogy to Equation (7). 
From this follows the following corollary, which gives us a first idea about how to define the moves in Projective Berlekamp:

Corollary 4.3. Each of the following sets of $m$-switchable subspaces generates the complete set of all m-switchable homotety-invariant patterns:

(i) The $B_{\langle v\rangle}:=\left\langle\{v\} \cup e_{\operatorname{ker}(v)}\right\rangle$ and $\left\langle e_{\operatorname{ker}(v)}\right\rangle$ with $v \in \mathbb{F}_{q}^{n}$ and $|\operatorname{ker}(v)| \geq m$.

(ii) The $U \leq \mathbb{F}_{q}^{n}$ of dimension $m$ or $m+1$ that contain at least $m$ coordinate axes.

(iii) The $U \leq \mathbb{F}_{q}^{n}$ of dimension $n-1$ or $n$ that contain at least $m$ coordinate axes, provided $r$ is non-zero and coprime to $q$.

Proof. We work here only with subspaces that contain at least $m$ coordinate axes and are $m$-switchable. Therefore, we only have to see that all $m$-switchable patterns are actually generated by these systems of $m$-switchable patterns.

In the case of the first system of patterns this is obvious. We know that the patterns $\bar{B}_{\langle v\rangle}$ with $|\operatorname{ker}(v)| \geq m$ form a generating system, and each $\bar{B}_{\langle v\rangle}$ (except $B_{\langle 0\rangle}$ ) is the difference of two elements, $B_{\langle v\rangle}$ and $\left\langle e_{\operatorname{ker}(v)}\right\rangle$, in our first system $\left(\bar{B}_{\langle 0\rangle}=B_{\langle 0\rangle}\right)$.

To prove that the second system is generating, it is enough to show that it generates all subspaces which contain $m$ coordinate axes, and, in particular, those in our first generating system. We show this by induction. Assume that we have already proven that subspaces of dimension $s$ and $s+1$ which contain $m$ coordinate axes are generated, and let $V$ be a subspace of dimension $s+2 \geq m+2$, containing $m$ coordinate axes. Select any subspace $V^{\prime} \leq V$ of dimension $s$, containing $m$ coordinate axes. Then $V^{\prime}$ and all spaces $H$ between $V^{\prime}$ and $V, V^{\prime}<H<V$, have dimension $s$ or $s+1$ and are generated by our system. It follows that $V$ is generated, because $V$ is a linear combination of these spaces, as

$$
q V^{\prime}+V=\sum\left\{H \mid V^{\prime}<H<V\right\},
$$

by Lemma 3.2, assuming that, w.l.o.g., $s+2=n, V=\mathbb{F}_{q}^{n}$.

The last generating property over $\mathrm{AB}_{r}^{n, m}$ follows with exactly the same inductive argument, just top down. If $V^{\prime}$ is a given $s$-dimensional subspace containing $m$ coordinate axes, then $q V^{\prime}$ is a linear combination of a fixed $V>V^{\prime}$ of dimension $s+2$ and all $H$ between $V^{\prime}$ and $V$, as above. To show that $V^{\prime}$, and not just $q V^{\prime}$, is generated, we need 1 to be a multiple of $q$, i.e., we need to play modulo $r$ (coprime to $q$ ).

With this, we are prepared to define Projective Berlekamp with Counter on the board

$$
\mathrm{PGC}_{n-1}\left(\mathbb{F}_{q}\right):=\left\{\langle v\rangle \mid v \in \mathbb{F}_{q}^{n}\right\}=\mathrm{PG}_{n-1}\left(\mathbb{F}_{q}\right) \uplus\{\langle 0\rangle\}
$$

and Projective Berlekamp on the projective space $\mathrm{PG}_{n-1}\left(\mathbb{F}_{q}\right)$ : 
Definition 4.4 (Projective Berlekamp). We write $\mathrm{PBC}^{n-1, m}=\mathrm{PBC}_{0}^{n-1, m}\left(\mathbb{F}_{q}\right)$ for $\mathrm{PBC}_{0}^{n-1, m}$ the Berlekamp $\left(\mathrm{PGC}_{n-1}\left(\mathbb{F}_{q}\right), \mathcal{P} \mathcal{M}_{m}\right)$ - called Projective Berlekamp with Counter and $\mathcal{P M}_{m}$ $m$-moves - where $\mathcal{P M}_{m}$ is the set of all subspaces $U^{1} \leq \mathrm{PBC}_{n-1}\left(\mathbb{F}_{q}\right)$, i.e. $U \leq \mathbb{F}_{q}^{n}$, which contain at least $m$ of the projective points $\left\langle e_{1}\right\rangle,\left\langle e_{2}\right\rangle, \ldots,\left\langle e_{n}\right\rangle$-called axes.

We write $\mathrm{PB}^{n-1, m}=\mathrm{PB}_{0}^{n-1, m}\left(\mathbb{F}_{q}\right)$ for the Berlekamp $\left(\mathrm{PG}_{n-1}\left(\mathbb{F}_{q}\right), \mathcal{P} \mathcal{M}_{m}\right)$ - called Projective Berlekamp with $m$-moves - where $\mathcal{P M}_{m}$ is defined as before, but we ignore the counter $\{0\}$ and view the moves $U^{1}$ as subsets of $\mathrm{PG}_{n-1}\left(\mathbb{F}_{q}\right)$, and as $0-1$ patterns $U^{1}: \mathrm{PG}_{n-1}\left(\mathbb{F}_{q}\right) \longrightarrow \mathbb{Z}$.

In the modulo $r$ case, with $\mathbb{Z}_{r}:=\mathbb{Z} / r \mathbb{Z}$ in the place of $\mathbb{Z}=: \mathbb{Z}_{0}$, we write $\mathrm{PBC}_{r}^{n-1, m}$, respectively $\mathrm{PB}_{r}^{n-1, m}$, with $r$ as index.

Since we derived our definition from Corollary 4.3, we see that $\mathrm{PBC}^{n-1, m}\left(\mathbb{F}_{q}\right)$ is basically the same as $\mathrm{AB}^{n-1, m}\left(\mathbb{F}_{q}\right)$ restricted to homotety-invariant patterns. We have:

Proposition 4.5. Let $r \in\{0,2,3,4, \ldots\}$. A homotety invariant pattern $U$ can be switched off in $\mathrm{AB}_{r}^{n, m}$ if and only if $U^{1}$ can be switched off in $\mathrm{PBC}_{r}^{n-1, m}$.

We also remark, that in the definition of $\mathrm{PBC}_{0}^{n-1, m}\left(\mathbb{F}_{q}\right)$ all $m$-moves $U^{1}$ contain the counter $\langle 0\rangle$. If we make an increasing move, we also increase the counter, if we make a decreasing move, we decrease the counter. This is why the counter is called counter.

Due to Corollary 4.3, it is further possible to restrict the set of moves $\mathcal{P M}_{m}$ to smaller generating sets. Consider the moves of projective dimension $m-1$ and $m$ in $\mathrm{PBC}^{n-1, m}$. In this case, we may even calculate how many moves of each dimension we need to switch off a switchable subspace $U^{1} \leq \mathrm{PG}_{n-1}$ of projective dimension $d$ :

Let $t_{1}$, respectively $t_{2}$, be the number of moves of projective dimension $m-1$, respectively $m$, where decreasing moves are counted negative. Then obviously

$$
t_{1}+t_{2}=-U(0)=-1 \text { and } t_{1} p^{m}+t_{2} p^{m+1}=-|U|=-p^{d+1} .
$$

The solution to this system of linear equations is

$$
t_{1}=p^{d-m}+p^{d-m-1}+\cdots+p \quad \text { and } \quad-t_{2}=p^{d-m}+p^{d-m-1}+\cdots+1 .
$$

One even can show that exactly $m$ of the $t_{2}$ "many" moves of projective dimension $m$ are coordinate subspaces $\left\langle e_{J}\right\rangle(|J|=m+1)$, provided that $U^{1}$ does not contain any axis $\left\langle e_{j}\right\rangle$.

In the important case $m=1$ (see [Sch1]), one can further ignore the $(m-1)$ dimensional, i.e. 0-dimensional, moves $\left\langle e_{j}\right\rangle$, and just unscrew the $n$ light bulbs $\left\langle e_{1}\right\rangle,\left\langle e_{2}\right\rangle, \ldots,\left\langle e_{n}\right\rangle$; they would be switchable individually, anyway. Therefore, in $\mathrm{PB}_{r}^{n-1,1}\left(\mathbb{F}_{q}\right)$ we obtain particularly nice switching rules: The lines that run trough at least one axis $\left\langle e_{j}\right\rangle$ are the switches (see Figure 3 at the end of the paper).

If, as usual, we interpret any pattern $U: \mathbb{F}_{q} \longrightarrow \mathbb{Z}$ as pattern $\mathbb{F}_{q} \backslash 0 \longrightarrow \mathbb{Z}$ on the smaller board $\mathbb{F}_{q}^{n} \backslash 0$, our basis $\bar{B}$ from above must contain a basis of the module of all patterns on this smaller board. We can prove the following analog to the both theorems 2.4 and 4.2 : 
Theorem 4.6. Let $f \in \mathbb{F}_{q}^{n}$, e.g. $f=0$. Then $\overline{\mathcal{B}} \backslash \bar{B}_{\langle f\rangle}$ is a basis of the module of all homotety-invariant patterns over $\mathbb{F}_{q}^{n} \backslash 0$. The $\mathbb{Z}$-submodule of m-switchable homotety-invariant patterns over $\mathbb{F}_{q}^{n} \backslash 0$ is saturated and has $\overline{\mathcal{B}}^{\geq m}:=\left\{\bar{B}_{\langle v\rangle} \mid v \in \mathbb{F}_{q}^{n}\right.$ with $\left.|\operatorname{ker}(v)| \geq m\right\}$ as a basis.

Proof. The rank of the free $\mathbb{Z}$-module of homotety-invariant patterns over $\mathbb{F}_{q}^{n} \backslash 0$ is obviously one smaller than the rank of the homotety-invariant patterns over $\mathbb{F}_{q}^{n}$. Therefore, it suffices to prove that $\overline{\mathcal{B}} \backslash \bar{B}_{\langle f\rangle}$ is a generating set. However, for vectors $u \neq 0$

$$
\begin{aligned}
\sum_{\langle v\rangle \in \mathrm{PGC}_{n-1}\left(\mathbb{F}_{q}\right)}(-1)^{|\operatorname{supp}(v)|} \bar{B}_{\langle v\rangle}(u) & =\sum_{v \in \mathbb{F}_{q}^{n}}(-1)^{|\operatorname{supp}(v)|} B_{0, v}(u) \\
& =\sum_{v: v_{j} \in\left\{0, u_{j}\right\}}(-1)^{|\operatorname{supp}(v)|}=\sum_{U \subseteq \operatorname{supp}(u)}(-1)^{|U|}=0,
\end{aligned}
$$

so that

$$
\sum_{\langle v\rangle \in \mathrm{PGC}_{n-1}\left(\mathbb{F}_{q}\right)}(-1)^{|\operatorname{supp}(v)|} \bar{B}_{\langle v\rangle} \equiv 0 \quad \text { on } \mathbb{F}_{q}^{n} \backslash 0 .
$$

Hence, $\overline{\mathcal{B}} \backslash \bar{B}_{\langle f\rangle}$ generates $\bar{B}_{\langle f\rangle}$ and $\overline{\mathcal{B}}$ and all the homotety-invariant patterns on $\mathbb{F}_{q}^{n} \backslash 0$.

To prove the second statement, we observe that $\overline{\mathcal{B}} \geq m$ is a subset of the basis $\overline{\mathcal{B}} \backslash B_{\langle f\rangle}$ if $f$ has full weight $(\operatorname{ker}(f)=\emptyset)$. Hence, $\overline{\mathcal{B}} \geq m$ is linearly independent, and obviously generates a submodule of $m$-switchable patterns. It even generates all $m$-switchable patterns. That is because, any $m$-switchable (homotety-invariant) pattern $U$ over $\mathbb{F}_{q}^{n} \backslash 0$ can be written as linear combination of $m$-moves. This linear combination can be viewed as $m$-switchable extension $\hat{U}: \mathbb{F}_{q}^{n} \longrightarrow \mathbb{Z}$ of $U: \mathbb{F}_{q}^{n} \backslash 0 \longrightarrow \mathbb{Z}$ to the full board $\mathbb{F}_{q}^{n}$. By Theorem 4.2, the homotety-invariant extension $\hat{U}$ is generated by $\overline{\mathcal{B}} \geq m$, so that $U$ itself is generated by $\overline{\mathcal{B}}^{\geq m}$, viewed as system of patterns on $\mathbb{F}_{q}^{n} \backslash 0$.

It would be desirable to find a "nice" complement to the submodule of $m$-switchable patterns, inside the $\mathbb{Z}$-module of homotety-invariant patterns on $\mathbb{F}_{q}^{n} \backslash 0$. One can show that, if $f$ is a fixed full weight vector, a complement is spanned by the linearly independent patterns $\langle v\rangle \backslash 0$ with $|\operatorname{ker}(v)|<m$ and $\langle v\rangle \nsubseteq\langle f\rangle$. Unfortunately, this complement depends on the choice of $f$, it is somehow asymmetric. A normal form based on the described compliment also would be asymmetric. In $\mathrm{PBC}^{n-1, m}$ the situation is different, here we can define a symmetric normal form $N^{m}$, based on the normal form $N_{0}^{m}$ of $\mathrm{AB}^{n, m}$, via

$$
N^{m}\left(U^{1}\right):=N_{0}^{m}(U)^{1} .
$$

We would like to use this definition also in $\mathrm{PB}^{n-1, m}$, but the expression $N_{0}^{m}(U)$ is not well defined over $\mathbb{F}_{q}^{n} \backslash 0$. If we just ignore the value $U(0)$ of 0 , then we treat the patterns $U$ and $U+c\{0\}$ as equal; $U+c\{0\}=U$ over $\mathbb{F}_{q}^{n} \backslash 0$. In this sense, $N_{0}^{m}(U+c\{0\})$ and $N_{0}^{m}(U)$ would be two normal forms to the same pattern. The normal form $N_{0}^{m}(U)$ for homotety-invariant patterns $U$ on $\mathbb{F}_{q}^{n} \backslash 0$ would be unique only up to a multiple of

$$
N_{0}^{m}(\{0\})=\underbrace{\{0\}}_{\substack{\equiv 0 \text { on } \\ \mathbb{F}_{q}^{n} \backslash 0}}-\sum_{v:|\operatorname{ker}(v)| \geq m}(-1)^{|\operatorname{supp}(v)|} B_{0, v}=\sum_{\substack{\langle v\rangle \in \operatorname{PGC}_{n-1}\left(\mathbb{F}_{q}\right) \\ \operatorname{ker}(v) \geq m}}(-1)^{|\operatorname{supp}(v)|} \bar{B}_{\langle v\rangle},
$$


which, in the special case $m=1$, equals $\pm\left(\mathbb{F}_{q} \backslash 0\right)^{n}$. Transferred to $\mathrm{PB}^{n-1, m}$, this means that we obtain a normal form $N^{m}\left(U^{1}\right)$ of a pattern $U^{1}: \mathrm{PG}_{n-1}\left(\mathbb{F}_{q}\right) \longrightarrow \mathbb{Z}$ via Definition (27), but it is only unique up to multiples of $N_{0}^{m}(\{0\})^{1}$. Practically, we can transform a given pattern $U^{1}: \mathrm{PG}_{n-1}\left(\mathbb{F}_{q}\right) \longmapsto \mathbb{Z}$ into this normal form $N^{m}\left(U^{1}\right)$ in a similar manner as we described it for $\mathrm{AB}^{n, m}$ after Theorem 2.4. At first, we have to switch off all projective points $\langle v\rangle$ with $|\operatorname{ker}(v)| \geq m$. This can be done step by step. In each step, we look for a "unfinished" point $\langle v\rangle$ with maximal $|\operatorname{ker}(v)|$, and use the composed move $\bar{B}_{\langle v\rangle}=\left\langle\{v\} \cup e_{\operatorname{ker}(v)}\right\rangle-\left\langle e_{\operatorname{ker}(v)}\right\rangle$ to switch it off. At the end, no projective point $\langle v\rangle$ with $|\operatorname{ker}(v)| \geq m$ is left,

$$
|\operatorname{ker}(v)| \geq m \Longrightarrow U^{1}(\langle v\rangle)=0
$$

and the resulting normal form will be uniquely determined up to multiples of $N_{0}^{m}(\{0\})^{1}$. An example is discussed at the end of this section, and illustrated in Figure 3.

If $q=2$ then every pattern is homotety-invariant and there is basically no difference between the projective and affine version of the game. The projective game $\operatorname{PB}^{n-1,1}\left(\mathbb{F}_{2}\right)$ without counter is quite uninteresting. Any pattern $U^{1}$ in $\operatorname{PB}^{n-1,1}\left(\mathbb{F}_{2}\right)$ can be switched off, since any pattern $U$ in $\mathrm{AB}^{n, 1}\left(\mathbb{F}_{2}\right)$ can be switched into a singleton $c\{0\}$, which is discarded in the projective game.

We want to study $\mathrm{PB}^{n-1, m}\left(\mathbb{F}_{q}\right)$ with $q \neq 2$. Since we removed the artificial counter, we will need additional assumptions to prove a statement similar to Proposition 4.5. We will see that, if $q \neq 2$, the property of being a linear subspaces $U \leq \mathbb{F}_{q}^{n}$ is strong enough. The main theorem about the maximal weight of the dual code will hold in this case as well. To prove this, we will need the following lemma about Affine Berlekamp; in which, for any pattern $U: \mathbb{F}_{q}^{n} \longrightarrow \mathbb{Z}$ and any vector $v \in \mathbb{F}_{q}^{n}$, the shifted pattern $\mathrm{M}_{v}(U)$ is defined pointwise via

$$
\mathrm{M}_{v} U(x+v):=U(x) \text { for all } v \in \mathbb{F}_{q}^{n}
$$

Since usually $n \neq 1$, this definition of $\mathrm{M}_{v}$ will not collide with our earlier definition of $\mathrm{M}_{\lambda}$, with $\lambda \in \mathbb{F}_{q} \backslash 0$. We provide:

Lemma 4.7. Let $q \neq 2, r \in\{0,2,3,4, \ldots\}$ and $c \in \mathbb{Z}_{r} \backslash 0$. For homotety-invariant patterns $U: \mathbb{F}_{q}^{n} \longrightarrow \mathbb{Z}_{r}$ the following switchability statements in $\mathrm{AB}_{r}^{n, m}$ hold:

(i) $U+c\{v\}$ cannot be switched off, for any choice of $v \in \mathbb{F}_{q}^{n}$ except possibly $v=0$.

(ii) $U+c\{0\}$ cannot be switched off, if $\mathrm{M}_{v}(U)=U$ for one vector $v \in \mathbb{F}_{q}^{n} \backslash 0$.

Proof. The first part follows mainly from Lemma 2.5. If $\operatorname{ker}(v)=\emptyset$, then

$$
N_{0}^{m}(c\{v\})=c\{v\} \neq 0
$$

and $c\{v\}$ cannot be switched off. Hence, any single-light pattern $c\{v\}$ cannot be switched off, even if $\operatorname{ker}(v) \neq \emptyset$, because switchability obviously does not depend on the relative position of a pattern on the board. Therefore,

$$
\operatorname{supp}\left(N_{0}^{m}(c\{v\}) \neq \emptyset\right.
$$


for any choice of $v \in \mathbb{F}_{q}^{n}$. Now, if $j \in \operatorname{supp}(v) \neq \emptyset$ then, for any $\lambda \in \mathbb{F}_{q} \backslash\{0,1\}$,

$$
x \in \operatorname{supp}\left(N_{0}^{m}(c\{v\})\right) \stackrel{2.5}{\Longrightarrow} \quad x_{j}=v_{j} \quad \Longrightarrow \quad \lambda x_{j} \neq v_{j} \stackrel{2.5}{\Longrightarrow} \lambda x \notin \operatorname{supp}\left(N_{0}^{m}(c\{v\})\right) .
$$

Thus, $N_{0}^{m}(c\{v\})$ is not homotety-invariant, if $q \neq 2$; but $N_{0}^{m}(U)$ is homotety-invariant, since $U$ is. Hence, $N_{0}^{m}(U+c\{v\})=N_{0}^{m}(U)+N_{0}^{m}(c\{v\})$ is not homotety-invariant and, in particular, not zero, i.e., $U+c\{v\}$ cannot be switched off.

In order to prove the second part, assume $U=\mathrm{M}_{v} U$, then $U+c\{0\}$ is switchable if and only if $\mathrm{M}_{v}(U+c\{0\})=U+c\{v\}$ is switchable, but we already have seen that this is not the case.

We also remark that any $m$-switchable patterns $U: \mathbb{F}_{q}^{n} \longrightarrow \mathbb{Z}$ obviously fulfills

$$
\sum_{v \in \mathbb{F}_{q}^{n}} U(v) \equiv 0 \quad\left(\bmod q^{m}\right)
$$

as any elementary $m$-move changes this sum by $\pm q^{m}$. (See [Sch1] for further invariants.) This would also lead to a simpler proof of some special cases of Lemma 4.7. However, we need the full generality of this lemma in order to show that $\mathrm{PB}^{n-1, m}\left(\mathbb{F}_{q}\right)$ behaves similar to $\mathrm{AB}^{n-1, m}\left(\mathbb{F}_{q}\right)$ (always provided that we study only linear subspaces $\{0\} \neq U \leq \mathbb{F}_{q}^{n}$, and $q \neq 2)$. We can prove:

Theorem 4.8. Let $q \neq 2$ and $r \in\{0,2,3,4, \ldots\}$. Let $U: \mathbb{F}_{q}^{n} \longrightarrow \mathbb{Z}_{r}$ be a homotetyinvariant pattern with $\mathrm{M}_{v}(U)=U$ for one vector $v \in \mathbb{F}_{q}^{n} \backslash 0$. Then $U$ can be switched off in $\mathrm{AB}_{r}^{n, m}$ if and only if its projective copy $U^{1}$ can be switched off in $\mathrm{PB}_{r}^{n-1, m}$.

Proof. If $U$ is switchable in $\mathrm{AB}_{r}^{n, m}$ then $U^{1}$ is switchable in $\mathrm{PB}_{r}^{n-1, m}$, since it is switchable even in $\mathrm{PBC}^{n-1, m}$, as we have seen in Proposition 4.5. Now assume that $U^{1}$ can be switched in $\mathrm{PB}_{r}^{n-1, m}$, then $U$ can be switched into a pattern $c\{0\}$ in $\mathrm{AB}_{r}^{n, m}$. Thus, $U-c\{0\}$ can be switched off. However, by Lemma 4.7, this is only possible for $c=0 \in \mathbb{Z}_{r}$, so that the switchability of $U$ in $\mathrm{AB}_{r}^{n, m}$ follows.

Using this connection to Affine Berlekamp, we can translate Theorem 3.3 into the projective language. We obtain:

Theorem 4.9. Let $q \neq 2$. For subspaces $U \leq \mathbb{F}_{q}^{n}, m \in\{1,2, \ldots, n-1\}$, and $r \in$ $\{2,3,4, \ldots\}$ not dividing $|U|$, the following are equivalent:

(i) $U^{\perp}$ has maximal weight at most $n-m$.

(ii) $U^{1}$ can be switched off in $\mathrm{PB}^{n-1, m}$.

(iii) $U^{1}$ can be switched off modulo $r$, in $\mathrm{PB}_{r}^{n-1, m}$.

Proof. This follows from Theorem 3.3 and Theorem 4.8. 
In both, Theorem 4.9 and Theorem 3.3, we assumed that $r$ does not divide $|U|$. It might look more natural to assume $r$ to be coprime to $q$, but our condition is more general. In general, one cannot drop a condition like this completely, as the following example in $P B_{3}^{2,1}\left(\mathbb{F}_{3}\right)(n=3, m=1, q=3, r=3)$ shall demonstrate. We examine the subspace $U:=\langle(1,1,1)\rangle^{\perp} \leq \mathbb{F}_{3}^{3}$. On one side, it has dual maximal weight $3 \not \leq n-m$, but, on the other side $U^{1}$ is still $m$-switchable modulo 3 , as the reader may check in the following picture:

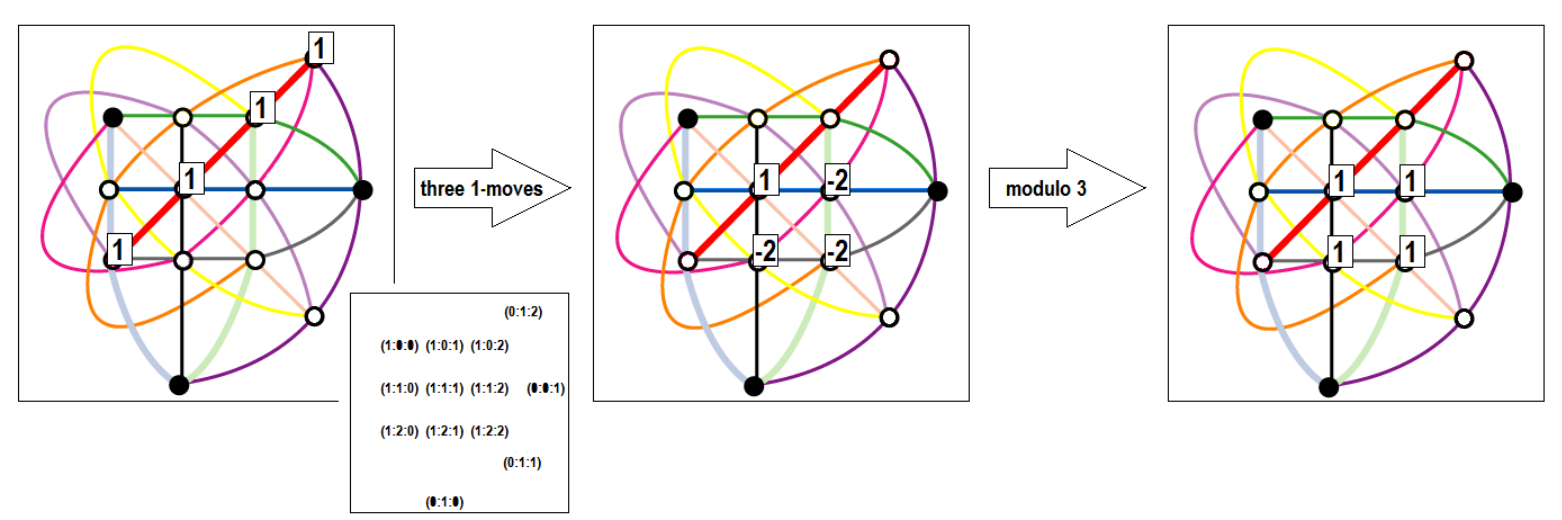

Figure 3: $U^{1}:=\left(\langle(1,1,1)\rangle^{\perp}\right)^{1} \leq \mathrm{PG}_{2}\left(\mathbb{F}_{3}\right)$ and its (up to multiples of $N_{0}^{m}(\{0\})^{1}$ unique) normal form $N_{0}^{1}(U)^{1}$, which is modulo 3 equal to $\left(\left(\mathbb{F}_{q} \backslash 0\right)^{3}\right)^{1}=-N_{0}^{1}(\{0\})^{1}$ and equivalent to the zero pattern $\emptyset$

\section{Acknowledgement:}

We want to thank Ayub Khan for his help. Thanks also go to the Abdus Salam School of Mathematical Sciences, where we finished the paper. Last but not least, the author gratefully acknowledges the support provided by the King Fahd University of Petroleum and Minerals under the project number IN100028.

\section{References}

[CaSt] J.Carlson, D. Stolarski: The Correct Solution to Berlekamp's Switching Game. Discrete Math. 287 (2004), 145-150.

[FiSl] P. C. Fishburn, N. J. A. Sloane: The Solution to Berlekamp's Switching Game. Discrete Math. 74 (1989), 263-290.

[MWS] F. J. MacWilliams, N. J. A. Sloane: The Theory of Error-Correcting Codes. North-Holland mathematical Library, Amsterdam 1978.

[RoVi] R. M. Roth, K. Viswanathan: On the Hardness of Decoding the Gale-Berlekamp Code. IEEE Trans. Inform. Theory, 54 (2008), 1050-1060.

[Sch1] U.Schauz: Colorings and Nowhere Zero Flows of Graphs in Terms of Berlekamp's Switching Game. Electron. J. Combin. 18/1 (2011), \#P65.

[Sch2] U.Schauz: Algebraically Solvable Problems: Describing Polynomials as Equivalent to Explicit Solutions. Electron. J. Combin. 15 (2008), \#R10. 